\title{
Consumer Scene Interactive Social Emotional Marketing: The Triple Dimension of Webcasting with Goods
}

\author{
Wang $\mathrm{Xia}^{1, \mathrm{a}}$, Kaderya $\cdot$ Arken $^{2, \mathrm{~b}^{*}}$ \\ ${ }^{1}$ School of Journalism and Communication, Xinjiang University, Urumqi, Xinjiang, China \\ ${ }^{2}$ School of Journalism and Communication, Xinjiang University, Urumqi, Xinjiang, China \\ a956211090@qq.com \\ b*956211090@qq.com
}

\begin{abstract}
With the development of livestreaming technology and the livestreaming industry, network anchors are no longer limited to obtaining income through livestreaming interaction with fans and receiving virtual gifts, but develop into livestreaming with hosts. Affected by the COVID-19 epidemic, live streaming has become an important way to drive sales growth. Traditional mainstream media and county-level local media have also joined the army of live streaming, realizing double harvest of social and economic benefits and becoming a new attempt of media integration development. Therefore, this article attempts from the construction of consumption scenarios, the formation of the interactive relationships and emotional experience three aspects to analyze the live webcast with the characteristics of the cargo, anchor created by users gathered in online shopping scene, recommended products in all forms, and based on the established connection and interaction with users of social relations, Promote users to be interested in the products recommended by anchors and generate consumption behaviors.
\end{abstract}

Keywords: Webcasting with Goods, Consumer Scene Interactive Social Emotional Marketing

\section{消费场景 互动社交 情感营销: 网络直播带货的三重维 度}

王霞 $1, \mathrm{a}$ 凯迪尔亚・艾尔肯 $2, \mathrm{~b}^{*}$

\author{
${ }^{1}$ 新疆大学新闻与传播学院, 乌鲁木齐, 新疆, 中国 \\ 2 新疆大学新闻与传播学院, 乌鲁木齐, 新疆, 中国 \\ a956211090@qq.com \\ b*956211090@qq.com
}

摘要:

随着直播技术和直播行业的发展, 网络主播早已不再局限于通过与粉丝用户直播互动、收取虚拟礼物等这一途 径获取收入, 而是发展为带货主播。受新冠肺炎疫情影响, 直播带货成为了带动销量增长的重要方式, 传统主 流媒体和县级地方媒体也纷纷加入直播带货的大军, 实现了社会经济效益双丰收, 成为了媒体融合发展的新尝 试。因此, 本文试图从消费场景的建构、互动社交关系的形成和情感体验三个方面来分析网络直播带货的特征, 主播通过将用户聚集到所创造的线上购物场景中，以各种形式推荐产品，并且基于与用户所建立的情感联系与 互动社交关系，促使用户对主播推荐的产品感兴趣并且产生消费行为。

关键词：网络直播带货 消费场景 互动社交 情感营销 


\section{1. 研究背景与文献回顾}

根据第 45 次《中国互联网络发展状况统计报告》 显示, 截至 2020 年 3 月, 我国网络直播用户规模达 5.60 亿，占网民整体的 $62.0 \%$ ，在 2019 年兴起并实 现快速发展的电商直播用户规模为 2.65 亿, 占网民 整体的 29.3\%。[1]随着年初新冠肺炎疫情的暴发, 全国经济停滞, 居民出入受限时, 网购便成为了人们 最直接的消费方式, 而网络直播带货作为一种新兴的 营销模式，在后疫情时代进入到了又一发展的新高 潮。

回顾文献发现, 相关研究包括有几类, 关于直播 带货的市场现状, 发展脉络, 以及媒介融合和现存问 题与挑战等方面的探究, 且大多数研究是从广告营 销, 传播策略, 消费结构转型等方面考虑直播带货对 社会经济的拉动作用, 鲜有文章专门探讨直播带货这 一传播行为的本质特征。例如有学者从直播电商的发 展历史、直播模式、传播理论以及发展现状、产业结 构等方面进行研究, 试图预测直播+电商的未来生态。 [2]还有学者从传播学角度出发, 提出网络直播带货 对媒体融合发展的启发, 例如学者赖雅红区分了传统 直播和网络直播的不同, 分析了直播带货的传播效 果。[3] 有研究认为直播带货成为传统媒体融合转型 突破口, 例如以央视为代表的主流媒体, 地方媒体等 与网红主播进行跨界合作进行公益传播, 实现扶贫助 农, 这不仅重塑了主流媒体影响力, 也是传统媒体与 互联网技术为代表的新媒体之间深度融合的有益探 索。[4] 此外还有研究从当前网络直播带货中存在的 问题分析入手, 尝试提出相应的对策以促进直播带货 健康良好发展。[5]

网络直播带货作为一种依托于移动互联网技术 的传播行为, 因此无论从营销模式和营销利润都给传 统的销售模式带来了巨大的冲击, 本文试图从消费场 景的建构、互动社交关系的形成和情感体验三个方面 来理解网络直播带货的特征。首先是消费场景的变 化, 由传统的线下场景变为线上场景, 其次是人与商 品的关系也发生了变化, 在直播的过程中建立了以消 费者为中心的新型互动社交关系, 而这种关系对于产 品来说是无形的资产, 是靠主播与消费者用户之间的 情感来维系。

\section{2. 消费场景建构: 现实空间和虚拟空间的弥合}

梅罗维茨将麦克卢汉 “媒介即信息” 的观点和戈 夫曼的 “拟剧理论” 结合起来, 提出了媒介情境论, 指出电子媒介正是通过改变人们的交往场合的方式 来影响人类传播的。彭兰认为, 移动时代场景的意义 大大强化, 移动传播的本质是基于场景的服务, 即对 场景（情境）的感知及信息（服务）适配。当越来越 多的信息与服务依赖场景这一变量时, 场景本身, 可 以成为信息组织、关系组织与服务组织的核心逻辑, 可以成为信息一关系一服务等几者连接的纽带, 进一 步, 场景本身可能成为移动平台的新入口。[6]直播
带货就是依赖于所建构的消费场景, 使有着类似消费 需求的不同用户聚集到此场景中, 并且适配相应的购 物信息和服务。

场景是吸引用户, 并为用户带来沉浸式体验的一 种社交与商业的情境。移动互联网时代, 依托网络直 播技术所构建的线上购物场景, 将无数个用户通过主 播彼此连接起来, 为其提供相应的产品信息和购物服 务, 并且建立了用户与主播, 用户与用户之间的情感 联系。网络带货直播间作为依靠移动平台技术构造的 消费场景, 它打破了时空限制的壁垒, 聚集了内容, 服务与关系为一体。直播间被打造成一个适应此次所 推销产品主题的购物环境, 使之画面呈现在有限的手 机屏幕里, 通过主播将一个个处于不同现实场景中的 用户聚集到主播间, 参与实时互动, 接收动态商品展 示, 形成更直观全方位的感官体验, 并且用户可以随 时随地进入或退出直播间。

网络直播带货所构建的移动化的消费场景塑造 了新的时空关系，弥合了现实空间与虚拟空间，具有 碎片化、即刻性、交互性，沉浸性的特点。直播带货 中虚拟空间与现实空间的缝隙不断弥合, 用户在并置 的时空关系中可以永远 “在场”。用户可以从头到尾 完整观看一次直播带货, 也可以随时进入和退出直播 间，而后者就导致进入直播间的用户是通过碎片式的 信息获取方式了解产品内容和信息, 主播限时限量抢 购的下单方式满足了用户即刻性的消费需求, 激起了 购物欲望。交互性和沉浸性不仅体现在主播与用户之 间的弹幕互动, 同时也受到主播的直播技巧和传播策 略的影响。此外，技术进步的驱动也增强了交互和沉 浸效果, 随着 VR 和 AI 等技术的进步和应用的普及, 直播带货所构造的消费场景将更加真实可感。

\section{3. 互动社交一建构主播与消费者间的强弱关 系}

网络直播带货要想达到营销目的，产生消费行 为, 仅仅搭建消费场景还是不够的, 营销过程中的核 心是主播与消费者之间的一种互动关系的建构, 恰恰 是这种互动关系使得产品拥有了大量的消费者, 而产 品本身则是这一关系的最后一个环节。因此, 网络直 播的另一个重要维度是通过建立以消费者为中心的 新型社交互动关系，主播作为消费者和商品之间连接 的纽带, 通过为用户提供信息服务以及产品推销, 使 消费者形成一定的购买意愿, 并将注意力转变为购买 力。

带货主播前期通过树立人设, 转发抽奖活动, 打 造口碑来圈粉, 与用户建立较稳定的互动社交关系。 近年来，“人设” 逐渐引延到娱乐产业中明星的批量 制造, 指自身的形象定位, 这种定位往往是积极正面 且深受大众喜爱的，加深大众对其的认可，从而达到 圈粉的目的。[7] 以网红带货主播李佳琪为例, 他以 专业的美妆知识, 每天不间断直播的 “敬业” 的工作 态度, 以及他良好的外在形象为其打造了 “口红一 
哥”、“带货达人” 等人设。此外李佳琦被认为兼具 男女特色，不像其他男性美妆博主过分阴柔，且又能 充当女性 “闺蜜” 提出购买意见, 与传统性别角色冲 突带来反差感, 以及跨越性别屏障的异样审美感受, 打破受众固有的认知框架, 能使受众印象深刻。[8] 并且利用了 “ $O M G$ ，买它买它买它！”等独特的语言 符号, 吸引了受众的注意力, 加深了对产品的印象。

此外, 主播在微博上不定期的转发抽奖送礼物或 现金的活动极大地调动了网友的参与度和积极性, 而 粉丝也为增大自己的中奖率会在微博转发评论中积 极进行互动回应博主, 努力成为 “铁粉” 。同时粉丝 的应援与推广也会为其赢得更多目标网友的注意力, 这些粉丝都将是网红博主潜在的消费者。因此, 主播 与用户间互动社交关系的强弱, 是消费行为是否产生 的重要原因。

\section{4. 情感体验：增强用户粘性的营销手段}

在直播带货中, 网络主播与用户间互动社交关系 的建立和维持是需要情感因素来维系的, 这与传统商 业行为中主要以价格和产品质量来维系关系并达到 营销目的是不同的。因此, 在直播带货中的情感维系 具体表现在发弹幕实时互动, 利用粉丝情感效应等。

带货主播不仅要了解和掌握产品的相关专业知 识与技能, 而且还要通过维持自身热度与人设, 注重 双向互动来增强用户粘性, 稳固与用户的的情感联 结, 才能使用户获得良好的情感体验, 从而增强购买 意愿, 为所推荐的产品消费买单。在直播过程中, 主 播通过弹幕同用户进行了实时互动, 无形之间产生了 情感纽带, 无论用户在观看过程中是否有过主动分享 或者互动的行为, 都在这场直播中获得了特殊的情感 体验。[9]

直播过程中的发 “弹幕” 功能, 让用户在直播间 感受到了心理和行动上的 “使用与满足”。[10]在直 播间，观看直播的用户通过发 “弹幕” 实时发表自己 的意见和看法, 以回应主播所制造的话题内容, 或是 与直播间里其他用户进行评论互动, 形成了多向的信 息表达与交流互动, 这使用户获得了一种虚拟的陪伴 感和认同感。正是基于对主播的情感依赖驱使下, 在 直播带货中唤起了用户对主播本身以及所推销产品 的注意力, 促使了进一步的消费行为, 因此用户在观 看直播带货时不仅获得了物质上的满足, 更是在主播 团队所营造的购物氛围、专业介绍、情境化互动等方 面获得了情感上的满足。

粉丝是对消费对象投入最多情感的群体, 不同于 普通消费者更加偏向实用功效和理性决策。 [11]粉 丝用户在直播间进行消费时，不仅仅是因为商品本身 的价值能够满足自身的物质需求, 更是出于对主播的 认同和追随的情感。粉丝用户在消费中倾注了感情, 此时消费行为不是为了实际需求的满足, 而是实现不 断被创造出来、被刺激起来的欲望的满足。
带货主播与流量明星合作, 正是擅用了情感能量 作为营销手段。新媒体时代的粉丝已经不满足于欣赏 偶像的音乐影视作品, 而是通过实际行动来表达对偶 像的喜爱和支持, 从最初的购买专辑、周边, 演唱会 和电影门票、再到现在的购买偶像代言的产品或同 款, 线下支援, 线上拉票等, 在当今 “饭圈” 中流行 着 “无消费不粉丝” 的口号, 实现了流量变现, 粉丝 经济的迅猛发展, 正是流量背后的粉丝购买力。正因 如此, 流量明星空降网红直播间的营销策略善用了粉 丝爱屋及乌的情感, 以此作为纽带建立与明星偶像之 间的情感沟通, 使粉丝对偶像的情感不自觉的转移到 相应的品牌和产品上, 产生消费行为。

情感互动在网络直播中通常作为维持主播与粉 丝用户之间关系的稳定剂。在直播中，自带流量的主 播通过与粉丝近距离的实时互动, 展示形象的同时推 销品牌和产品, 带动了更多的粉丝消费。因此直播带 货的特征之一就是依靠情感营销的模式, 使粉丝情感 与经济回报相挂钩, 利用长期积累的人脉和粉丝用户 资本进行流量变现。

\section{5. 结论}

基于各界带货主播所营造的场景策略, 情感动员 和社交关系是实现用户购买行为转化的核心动力, 因 此消费场景的建构，主播和用户间的强弱关系互动以 及情感营销是网络直播带货的本质特征。直播带货对 传统媒体经营模式转型是一种新的尝试, 媒体融合应 该更加重视直播带货的传播机制和本质特征, 创新媒 体传播方式, 激发受众参与传播的积极性, 从而形成 传播价值、用户价值、社会价值的多赢局面, 使直播 带货这一线上营销环境健康有序发展, 真正成为刺激 国民消费和拉动经济的催化剂。

\section{REFERENCES}

[1] The 45th CNNIC Statistical Report on China's Internet Development,2020(04). http://www.cac.gov.cn/2020-04/27/c_15895354703 78587.html

[2] Zhao zizhong,Chen lianzi.(2020)Communication Theory, Development Status, Industrial Structure and Reflection of Live Streaming E-commerce.J.China Broadcast.,11-18.

[3] Lai yahong.(2020)The Inspiration of The Development of Webcasting and Media Convergence Based on The Perspective of Communication.J.Journal of News Research .,132-133.

[4] Mei Qian.(2020)Barriers, Problems and Effects of media convergence -- Based on the analysis and thinking of "Xiao Zhu Pei Qi" Webcasting with goods.J.New Media Research.,81-83.

[5] Lin ZiYi,Fan Li.(2020)Problems and 
Countermeasures in the Current Webcasting with Goods.J..New Media research.,49-51+58

[6] Pen Lan.(2015)Scene: New Elements of Media in the Mobile Age,J.Journalism Review.3.

[7] Chen Huan.(2019)A Probe into the Characteristics and Construction Strategies of the Star's "Personalization" From the Dramaturgical Theory.J.Journal of News Research .,48-49.

[8] Xu JiaMin.(2019) Communication Characteristics and Audience Psychology of Short Beauty Videos: Based on The Case of Li JiaQi.J.New Media Research.,51-53.

[9] Wang YanLin,Liu Ke.(2019)Resonance Effect of Network Broadcast: Group Loneliness, Virtual Emotion and Consumer Identity.J.Modern Communication.J.Modern Communication.,26-29

[10] Zhou YiChen.(2020)Analysis On the Phenomenon of Network Broadcast From the Perspective of Scene Communication.J.Research On Transmission Competence., 191+193

[11]Zhou YiJin,Huang YingJia,Liang ShiMin.(2020)The Four-fold Relationship Between "Fans" and "Be fans" : A Study On the Economy of Living Broadcast Fans with Goods.J.News and Writing,29-35. 